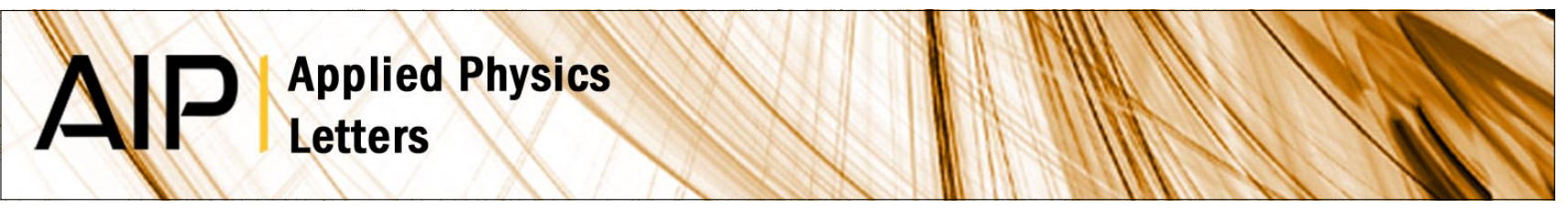

\title{
High-speed tapping mode imaging with active $Q$ control for atomic force microscopy
}

T. Sulchek, R. Hsieh, J. D. Adams, G. G. Yaralioglu, S. C. Minne et al.

Citation: Appl. Phys. Lett. 76, 1473 (2000); doi: 10.1063/1.126071

View online: http://dx.doi.org/10.1063/1.126071

View Table of Contents: http://apl.aip.org/resource/1/APPLAB/v76/i11

Published by the American Institute of Physics.

\section{Related Articles}

Bias controlled capacitive driven cantilever oscillation for high resolution dynamic force microscopy Appl. Phys. Lett. 102, 073110 (2013)

Friction measurement on free standing plates using atomic force microscopy

Rev. Sci. Instrum. 84, 013702 (2013)

A correlation force spectrometer for single molecule measurements under tensile load

J. Appl. Phys. 113, 013503 (2013)

Compact metal probes: A solution for atomic force microscopy based tip-enhanced Raman spectroscopy Rev. Sci. Instrum. 83, 123708 (2012)

Note: Radiofrequency scanning probe microscopy using vertically oriented cantilevers

Rev. Sci. Instrum. 83, 126103 (2012)

\section{Additional information on Appl. Phys. Lett.}

Journal Homepage: http://apl.aip.org/

Journal Information: http://apl.aip.org/about/about_the_journal

Top downloads: http://apl.aip.org/features/most_downloaded

Information for Authors: http://apl.aip.org/authors

\section{ADVERTISEMENT}

\section{AIP Applied Physics Letters}

\section{EXPLORE WHAT'S NEW IN APL}

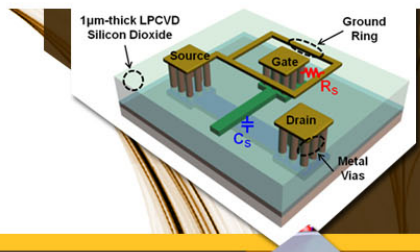

SURFACES AND INTERFACES

Focusing on physical, chemical, biological structural, optical, magnetic and electrical properties of surfaces and interfaces, and more.. 


\title{
High-speed tapping mode imaging with active $Q$ control for atomic force microscopy
}

\author{
T. Sulchek, ${ }^{\text {a) }}$ R. Hsieh, J. D. Adams, G. G. Yaralioglu, S. C. Minne, and C. F. Quate \\ E. L. Ginzton Laboratory, Stanford University, California 94305-4085 \\ J. P. Cleveland \\ Asylum Research, 601 Pine Avenue, Santa Barbara, California 93117
}

\author{
A. Atalar \\ Bilkent University, Ankara, 06533, Turkey \\ D. M. Adderton \\ NanoDevices Inc., 516 E. Gutierrez, Suite E, Santa Barbara, California 93103
}

(Received 2 December 1999; accepted for publication 19 January 2000)

\begin{abstract}
The speed of tapping mode imaging with the atomic force microscope (AFM) has been increased by over an order of magnitude. The enhanced operation is achieved by (1) increasing the instrument's mechanical bandwidth and (2) actively controlling the cantilever's dynamics. The instrument's mechanical bandwidth is increased by an order of magnitude by replacing the piezotube $z$-axis actuator with an integrated zinc oxide $(\mathrm{ZnO})$ piezoelectric cantilever. The cantilever's dynamics are optimized for high-speed operation by actively damping the quality factor $(Q)$ of the cantilever. Active damping allows the amplitude of the oscillating cantilever to respond to topography changes more quickly. With these two advancements, $80 \mu \mathrm{m} \times 80 \mu \mathrm{m}$ high-speed tapping mode images have been obtained with a scan frequency of $15 \mathrm{~Hz}$. This corresponds to a tip velocity of 2.4 mm/s. (c) 2000 American Institute of Physics. [S0003-6951(00)02811-4]
\end{abstract}

The atomic force microscope (AFM) has proven to be a useful tool for imaging a wide range of materials in a wide set of environments. Intermittent contact mode, or tapping mode, imaging was first introduced by Zhong et al. ${ }^{1}$ It has become the dominant mode of AFM imaging because it reduces lateral forces between the tip and sample. ${ }^{2}$

The cantilever driven at its resonant frequency will achieve a free air amplitude determined by the drive amplitude, the spring constant, and the quality factor of the cantilever's resonance $(Q)$. As the cantilever is brought into contact with the sample, the surface will limit the oscillatory motion. The amplitude is typically $10-100 \mathrm{~nm}$ and is measured with a split photodetector. The root-mean-square (rms) amplitude of the cantilever is kept constant with a feedback loop that controls the vertical distance between the tip and the sample. In most AFMs, the feedback loop controls a conventional piezotube such that when the sample topography causes the cantilever's rms amplitude to change, the piezotube will extend or contract to restore the cantilever's original rms value.

While the tapping mode AFM allows nanometer scale resolution with negligible frictional forces, it is encumbered by slow imaging speed. The scan speed of typical tapping mode AFMs is limited in part by the resonant frequency of the piezotube and in part by the time it takes for the oscillating cantilever to change amplitude. (Other factors play a role in the tapping mode imaging speed, such as the bandwidth of the rms to dc converter, but these are secondary). For most samples, these constraints limit the tapping mode AFM's scan speed to a few tens of microns per second. At this speed a single, moderately sized $512 \times 512$ pixel image will take several minutes to acquire.

${ }^{a)}$ Electronic mail: sulchek@stanford.edu
We have increased the tapping mode imaging rate with two improvements. First, a faster $z$-axis actuator is integrated onto the cantilever. Second, an active damping circuit is applied to increase the speed at which the cantilever can respond.

Several groups have succeeded in fabricating integrated piezoelectric bimorph actuators onto cantilevers. ${ }^{3-6}$ The bimorph displaces the tip vertically when a voltage is applied across the piezoelectric material. This type of cantilever in contact mode allows scan speeds up to $1 \mathrm{~cm} / \mathrm{s}^{6}{ }^{6}$

In other approaches for high-speed tapping mode imaging, Paloczi et al. ${ }^{7}$ have taken advantage of the low $Q$ and high resonant frequency of small cantilevers in liquid to image with scan speeds of $52 \mu \mathrm{m} / \mathrm{s}$ without the use of feedback. Ookubo et al. $^{8}$ obtained a $225 \mu \mathrm{m} / \mathrm{s}$ speed by combining the feedback signal and the error signal into a composite topographic signal. This method can indeed increase speed, but it does so at the expense of increased tip/sample force.

Here, we use a zinc oxide $(\mathrm{ZnO})$ piezoelectric actuator to both drive the cantilever at its resonant frequency and to provide $z$ actuation. The cantilever has been described previously. ${ }^{9}$ In Fig. 1, we compare the response of the integrated $\mathrm{ZnO}$ actuator with that of a typical piezotube actuator. ${ }^{10}$ The resonant frequency of the $z$ actuator marks the point of $180^{\circ}$ phase shift with the drive $\left(90^{\circ}\right.$ at the resonant frequency and $90^{\circ}$ from the feedback integrator). To avoid instability, the maximum gain of the feedback loop is limited such that the response at this frequency is less than one. Therefore, the $z$-actuator's resonant frequency sets an upper limit on imaging bandwidth. The addition of the integrated $\mathrm{ZnO}$ actuator increases the resonant frequency by nearly a factor of 40 over the piezotube.

In tapping mode, the scanning speed is additionally constrained by (1) a small maximum error signal which limits 


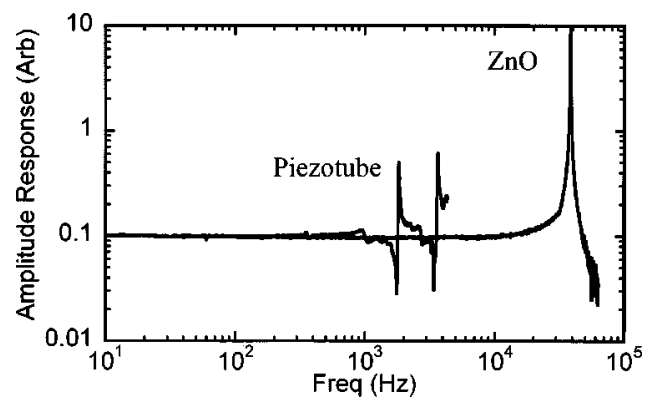

FIG. 1. Amplitude response of a $\mathrm{ZnO}$ cantilever and a conventional piezotube. The mechanical response of the $\mathrm{ZnO}$ is 40 times faster than that of the piezotube.

the feedback's output, and (2) the cantilever's high $Q$ which results in long time constants for changes in error signal. The error signal is defined as the setpoint, $a_{\mathrm{SP}}$ (the desired tapping amplitude), minus the rms amplitude, $a_{\text {rms }}$ (the actual tapping amplitude). Typically, the setpoint is chosen to be slightly below the free air amplitude of the cantilever. This ensures that the tip is tapping lightly on the sample. A property of tapping mode that limits the speed is the magnitude of the error signal. For accurate imaging, the signal should be proportional to the change in topography, but this is not always true. For the case where the tip encounters a sufficiently large upward step, the cantilever's amplitude will decrease until the oscillation is completely quenched. After this, the error signal becomes saturated at $a_{\mathrm{SP}}$. If the sample contains a sharp downward step, the sample will no longer impede the oscillation, and the cantilever amplitude will increase and saturate at the free air amplitude, $a_{\text {free }}$.

Since the setpoint is generally chosen to be close to the free air amplitude, the maximum error signal on a downward step is necessarily small. This limited error signal constrains the feedback loop to a slow response (in comparison to an upward step). In general, the speed of tapping mode imaging can be increased by simply using a smaller setpoint since this allows a larger error signal. However, this strategy requires a stronger tapping force which is undesirable.

When scanning over a downward step, a cantilever initially operating with an amplitude of $a_{\mathrm{SP}}$ (an error signal of zero), will ring up to a maximum amplitude of $a_{\text {free }}$ (a negative error signal of $a_{\mathrm{SP}}-a_{\text {free }}$ ). The $Q$ of an oscillating system is defined as $2 \pi$ times the mean stored energy divided by the work per cycle. ${ }^{11}$ For a high $Q$ cantilever driven at resonance, the error signal during a transient (resulting from a step) follows an exponential path with a time constant inversely proportional to $Q$. The tapping mode error signal will be delayed in response as the error signal grows to an appreciable value. For a cantilever system with a $Q$ of several hundred and a resonant frequency of 100 kilohertz, this ring-up can take more than a millisecond. In order to achieve nanometer resolution on samples with sharp features, this delay will limit the scan speed to a few microns per second.

Our solution to this problem is to use active damping to change the cantilever's apparent $Q$. Mertz et al. ${ }^{12}$ developed a system for actively damping an AFM cantilever to speed up the system's mechanical transients. Their system used a thermal bimorph actuator to apply the active control. Garbini et al. ${ }^{13}$ extended this work by designing a controller for active modification of cantilever dynamics to improve the sta-
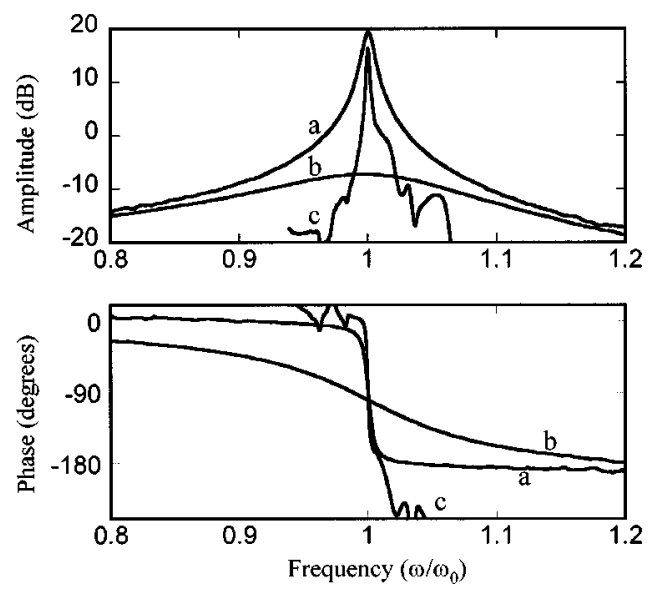

FIG. 2. Amplitude and phase responses of a $\mathrm{ZnO}$ cantilever and a commercial Si cantilever. The frequency axis has been normalized in units of $\omega_{0}$. Traces (a) and (b) show the resonance of the $\mathrm{ZnO}$ cantilever and the $\mathrm{ZnO}$ cantilever with active damping respectively. In this case, the cantilever is damped from a $Q$ of 150 to a $Q$ of 7. Trace (c) shows a typical resonance of a silicon cantilever driven with a commercial piezoslab. Because the actuator is external to the cantilever, the amplitude and phase show significant deviation from an ideal second order response. This is particularly a problem if $Q$ damping is attempted.

bility of magnetic force microscopy. Bruland et al. ${ }^{14}$ used a similar control system to actively control an ultrasoft magnetic cantilever with an external field.

Our active damping is accomplished with a feedback circuit. We can understand this technique by examining the equation of motion for the oscillating cantilever: $F=m \ddot{z}$ $+b \dot{z}+k z$ where $m$ is mass, $z$ is deflection, $b$ is damping factor, $k$ is spring constant, and $F$ is the cantilever drive. The derivatives are with respect to time. $Q$ is inversely proportional to the damping factor, $b$. For active damping of this system, we use the $\mathrm{ZnO}$ actuator to add a force that is proportional to the velocity of the cantilever for frequencies in the imaging bandwidth. The AFM measures the deflection of the cantilever, $z$, and thus is converted to velocity, $\dot{z}$, with an electronic differentiator or phase shifter. We then add this signal back into the drive to gain full control over the $Q$, and the transient response.

Trace (a) in Fig. 2 shows the amplitude and phase response of a $\mathrm{ZnO}$ cantilever at resonance with a resonant frequency of $46 \mathrm{kHz}$ and a $Q$ of 150 . In trace (b), we show the response is damped by a factor of 20 . Active damping allows the cantilever to reach its free air amplitude faster, and this reduces the delay associated with cantilever ring up. The $\mathrm{ZnO}$ cantilever is uniquely suited for active damping when compared to the response of a typical cantilever driven by a piezoslab shown in trace (c). In most AFMs, cantilevers are excited with a piezoslab located on a cantilever holder. The mechanical connection in this arrangement creates spurious responses for both amplitude and phase. These deviations from the ideal system make it difficult to stabilize and maintain active control.

In Fig. 3, we show the effect of active control on the cantilever ring-up time. There we display the error signal versus time when the cantilever suddenly loses contact with the surface. These results were obtained by tapping on a bare silicon sample that was quickly retracted at $t=0 .{ }^{15}$ In Fig. 3, trace (b) is the error signal for a cantilever with no active 


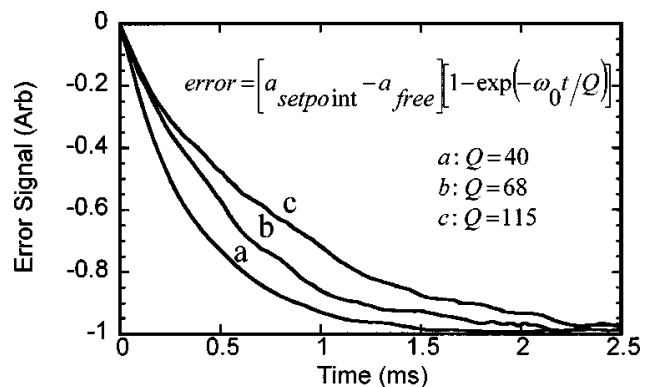

FIG. 3. Traces show the error signal versus time when the cantilever suddenly loses contact with a surface. Trace (b) is the error signal for a cantilever with no active control. The resonance in trace (a) is damped by a factor of 2. The resonance in trace (c) is enhanced by a factor of 2 . These curves were fit to an exponential and solved for $Q$.

control. Reducing the apparent $Q$ by a factor of 2 with active damping produces the faster ring-up time shown in trace (a). We can also increase the apparent $Q$ by reversing the gain of the active damping circuit. Trace (c) shows the slower response when the $Q$ is increased by a factor of 2 (although the increased $Q$ slows down imaging, it is useful to increase the range of the attractive regime ${ }^{16}$ ). We extracted the change in the apparent value of $Q$ by fitting the error signal to an ex- (a) Piezotube
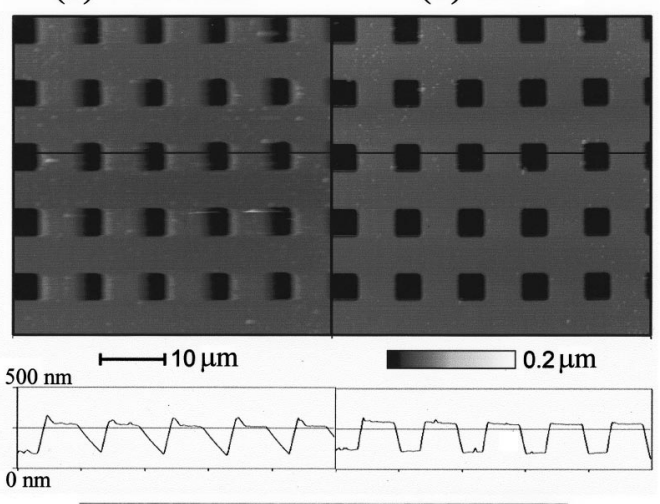

(c)

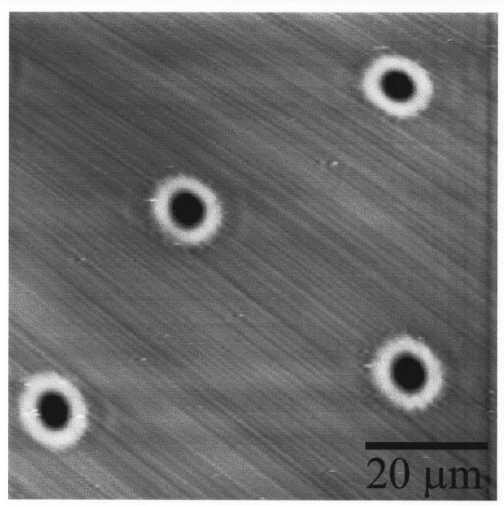

FIG. 4. (a) and (b) show images of a grating taken at a $5 \mathrm{~Hz}$ scan rate with equal tapping amplitudes, setpoints, and optimized gains. The $z$ actuator in (a) is a piezotube and in (b) is an actively damped $\mathrm{ZnO}$ cantilever. The line traces shows the piezotube's inability to effectively follow topography at these speeds. (c) shows an image of a hard drive disk using both fast $\mathrm{ZnO}$ actuator and active damping. The image scan size is $80 \mu \mathrm{m}$ and the scan rate is $15 \mathrm{~Hz}$, putting the imaging speed at $2.4 \mathrm{~mm} / \mathrm{s}$. This high-resolution image reveals the polishing of the disk and the laser zone texturing and has a $z$ range of $80 \mathrm{~nm}$. The image was taken in just over $17 \mathrm{~s}$. The image has been filtered to remove the vertical resonances of the $x y$ scanner caused when driven at a high rate. ponential. All of the $Q$ values are smaller than those observed in free air, but correspond to $Q$ measurements taken at $100 \mathrm{~nm}$ above the surface. This decrease in $Q$ when close to the surface is due to squeeze film damping between the cantilever and the sample. ${ }^{17}$

In Fig. 4, we show an image and a line trace of a grating taken with both the standard tapping mode system (a), and with the $\mathrm{ZnO}$ cantilever under active control (b). Both images were taken with a scan rate of $5 \mathrm{~Hz}$, with equal tapping amplitudes, setpoints, and optimized gains. The image taken with the piezotube as the $z$-axis actuator does not follow topography, and tends to become unstable when changes in height were encountered. However, the scan with the actively damped $\mathrm{ZnO}$ cantilever faithfully reproduces the sample topography. This is evident from the line traces. Figure 4(c) shows a $2.4 \mathrm{~mm} / \mathrm{s}$ image of a laser textured landing zone on a hard disk using both the fast $\mathrm{ZnO}$ actuator and active damping.

By combining the benefits of a fast $z$-axis actuator and active control of the cantilever dynamics, we can increase the speed and decrease the recording time for tapping mode images by more than an order of magnitude. Images of smaller size could be acquired faster, which may allow for fast, realtime imaging. The integrated actuator has been used with arrays of cantilevers operating in parallel, ${ }^{18}$ and now with active damping we should be able to achieve large scale imaging with minimum sample damage.

The authors would like to credit Jim Zesch for his work depositing $\mathrm{ZnO}$ films. S. C. M. and D. M. A. acknowledge support from NSF No. DMI-9903522 and DARPA DAAH01-00-C-R014. This work is supported by the Defense Advanced Research Projects Agency.

${ }^{1}$ Q. Zhong, D. Imniss, K. Kjoller, and V. B. Elings, Surf. Sci. 290, L688 (1993).

${ }^{2}$ S. H. Leuba, G. Yang, C. Robert, B. Samori, K. van Holde, J. Zlatanova, and C. Bustamante, Proc. Natl. Acad. Sci. USA 91, 11621 (1994).

${ }^{3}$ S. Akamine, T. R. Albrecht, M. J. Zdeblick, and C. F. Quate, IEEE Electron Device Lett. 10, 490 (1989).

${ }^{4}$ T. Itoh and T. Suga, in The Proceedings of Transducers '95, Stockholm (Royal Swedish Academy of Engineering Science, 1995), Vol. IV A, p. 632.

${ }^{5}$ T. Fujii, S. Watanabe, M. Suzuki, and T. Fujiu, J. Vac. Sci. Technol. B 13, 1119 (1995).

${ }^{6}$ S. R. Manalis, S. C. Minne, A. Atalar, and C. F. Quate, Rev. Sci. Instrum. 67, 3294 (1996)

${ }^{7}$ G. T. Paloczi, B. L. Smith, P. K. Hansma, D. A. Walters, and M. A. Wendman, Appl. Phys. Lett. 73, 1658 (1998).

${ }^{8}$ N. Ookubo and S. Yumoto, Appl. Phys. Lett. 74, 2149 (1999).

${ }^{9}$ S. C. Minne, G. Yaralioglu, S. R. Manalis, J. D. Adams, J. Zesch, A. Atalar, and C. F. Quate, Appl. Phys. Lett. 72, 2340 (1998).

${ }^{10}$ Dimension 3000, Digital Instruments, Santa Barbara, CA 93117.

${ }^{11}$ R. Feynman, The Feynman Lectures on Physics (1963), Vol. 1, p. 24-2.

${ }^{12}$ J. Mertz, O. Marti, and J. Mlynek, Appl. Phys. Lett. 62, 2344 (1993).

${ }^{13}$ J. L. Garbini, K. J. Bruland, W. M. Dougherty, and J. A. Sidles, J. Appl. Phys. 80, 1951 (1996).

${ }^{14}$ K. J. Bruland, J. L. Garbini, W. M. Dougherty, and J. A. Sidles, J. Appl. Phys. 83, 3972 (1998).

${ }^{15}$ This exponential increase in error signal is also observed when imaging downward steps.

${ }^{16}$ B. Anczykowski, J. P. Cleveland, D. Kruger, V. Elings, and H. Fuchs, Appl. Phys. A: Solids Surf. 66, S885 (1998).

${ }^{17}$ F. M. Serry, P. Veuzil, R. Vilasuso, and G. J. Maclay, in Proceedings of the Second International Symposium on Microstructures and Microfabricated Systems, Chicago (Electrochem. Soc., Pennington, NJ, 1995), p. 83.

${ }^{18}$ S. C. Minne, P. Flueckiger, H. T. Soh, and C. F. Quate, J. Vac. Sci. Technol. B 13, 1380 (1995). 